\title{
Tissue composite graft lacking sinuses may well prone to develop endocarditis post aortic root replacement: An untested hypothesis
}

\author{
Maqsood M. Elahi ${ }^{1}$, Dennis $\mathrm{Ho}^{2}$, Kenton J. Zehr ${ }^{1}$ \\ 1. Division of Cardiothoracic Surgery, Department of Surgery, Scott \& White Memorial Hospital, USA. 2. Division of \\ Cardiovascular Anesthesiosology, Department of Anesthesiology \& Texas A \& M Health Science Center, Scott \& White \\ Memorial Hospital, USA.
}

Correspondence: Maqsood Elahi. Address: Division of Cardiothoracic Surgery, Department of Surgery, Scott \& White Memorial Hospital, USA. Telephone: 1-254-724-7543. Email: manzoor_elahi@hotmail.com.

Received: November 6, 2012

Accepted: March 5, 2013

Online Published: April 22, 2013

DOI : $10.5430 / j b g c . v 3 n 3 p 49$

URL: http://dx.doi.org/10.5430/jbgc.v3n3p49

\begin{abstract}
A 72 year-old female with acute prosthetic aortic root endocarditis and systolic congestive heart failure underwent a homograft revision within 6-months. Initially she underwent root replacement with a straight composite graft for a severely stenotic aortic valve and root dilatation. Over a 6-month period, she developed a progressively audible holosystolic murmur. Echocardiography demonstrated a vibrating stuttering of the prosthetic valve cusps on M-mode. This was exaggerated with aortic root endocarditis vegetations on cusps. We hypothesize that this repetitive severe turbulent flow related to a Venturi effect damaged the straight tube graft-aortic root and prosthetic valve cusps in our case.
\end{abstract}

\section{Key words}

2D-Echo, M-mode Echo, Stentless bioprosthetic valve, Venturi effect, Dacron tube graft, Endocarditis

\section{I ntroduction}

The sinuses around the cusps are well known for their ability to help absorb aortic root pressure, especially during diastolic phase to prevent damage to aortic root area. They may also help protect the aortic root from damage of high velocity jet during systolic cycle if there is a supravalvular obstruction downstream. We hypothesize that patient with straight Dacron tube graft used for repairing the aortic root aneurysm with no sinuses around cusps are more likely to develop endocartitis of aortic root from venturi effect across the cusps.

\section{Case report}

A 72 year-old female with an aortic bioprosthetic valve and root endocarditis presented with acute systolic congestive heart failure. She underwent a redo aortic root replacement (ARR) with a homograft within 6 months of her primary operation, when earlier the patient underwent ARR with a composite graft created by sewing a $23-\mathrm{mm} 3 \mathrm{~F}$ stentless equine pericardial prosthesis into a 24-mm straight Dacron tube graft; coronary artery bypass graft (CABG) to right coronary 
artery; and replacement of the ascending aorta together with proximal hemiarch for severely stenotic aortic bicuspid valve, aortic root dilatation and aortic ascending aneurysm.

Over a six-month period after her initial operation, she developed a progressive high pitch audible holosystolic murmur, which ultimately became 6/6 heard throughout her precordium. On echo per M-mode, a high frequency vibrating stuttering of the prosthetic valve cusps were prominently seen. The patient also developed spondylodiskitis of L2-L3 and osteomyelitis confirmed by a positive culture for coagulase-negative staphylococcus from biopsy. Transesophageal echo (TEE) performed right after patient's admission demonstrated moderately reduced systolic function with estimated ejection fraction of $35 \%-40 \% ; 2$ mild eccentric jets of mitral regurgitation quantitatively assessed by vena contracta method; an aliasing eccentric jet crossing the aortic valve hitting the aortic root during the systolic phase of cardiac cycle (see Figure 1a). The echo showed increased echogenicity of the soft tissue surrounding the aortic root, indicating possible thrombus and vegetation formation. Figure $1 \mathrm{~b}$ shows a high frequency fluttering of the prosthetic aortic valve leaflets throughout the systolic phase per M-mode prior to the redo operation, that accounts for a prominent high pitch "buzzing" murmur sound on physical exam across the heart precordium. 2-D echo with color Doppler showed a mosaic color pattern between the equine aortic cusps during systolic phase indicating a high turbulent flow across the valve (see Figure 2a). Repeat echo (M-mode) after redo surgery showed fluttering of bioprosthetic aortic leaflets, but it has a much finer characteristic with decreasing frequency (see Figure-2b).

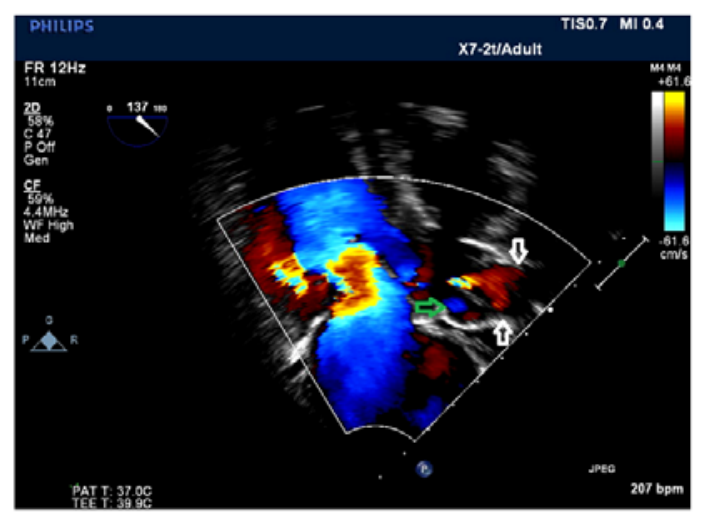

Figure 1. (a) TEE midesophageal long axis view of the aortic valve with color Dopper pattern. The turbulent high velocity flow divides into 2 jets as shown by the white arrows hitting the aortic root in opposite directions. The green arrow shows the color flow pattern of blood into the stretched tubular graft area below which may play a role in absorbing increasing pressure from the jet

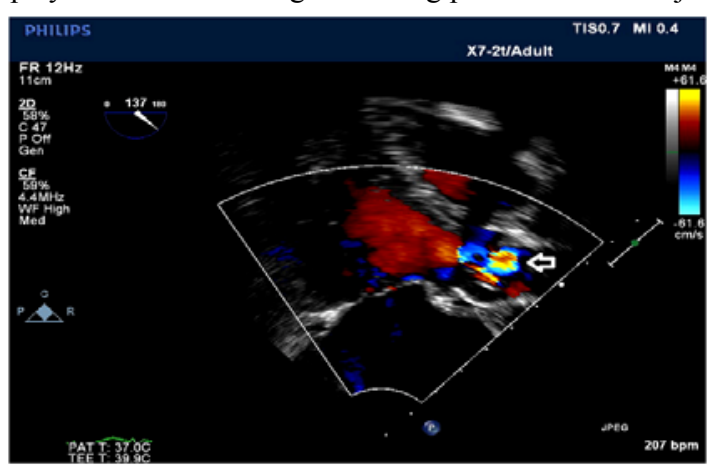

Figure 2. (a) TTE parasternal long axis view with color Doppler pattern. The white arrow shows a mosaic color pattern, which indicates a high turbulent flow between the aortic cusps during systolic phase of cardiac cycle

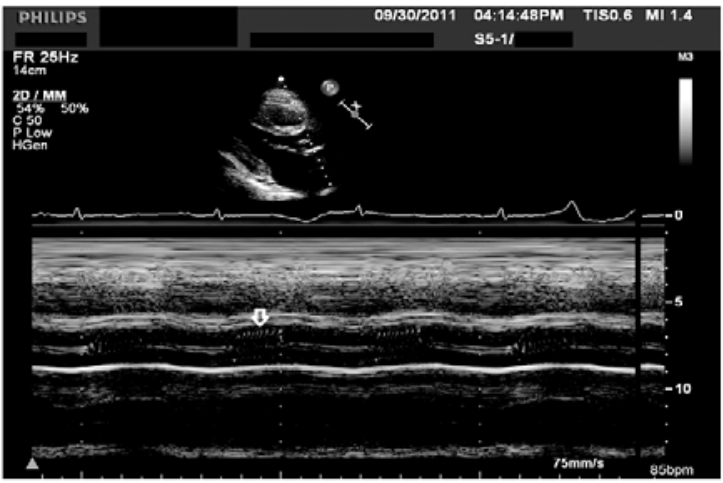

Figure 1. (b) TTE parasternal long axis view with M-mode. The white arrow indicates a high frequency holosystolic fluttering of aortic cusps prior to redo aortic root replacement

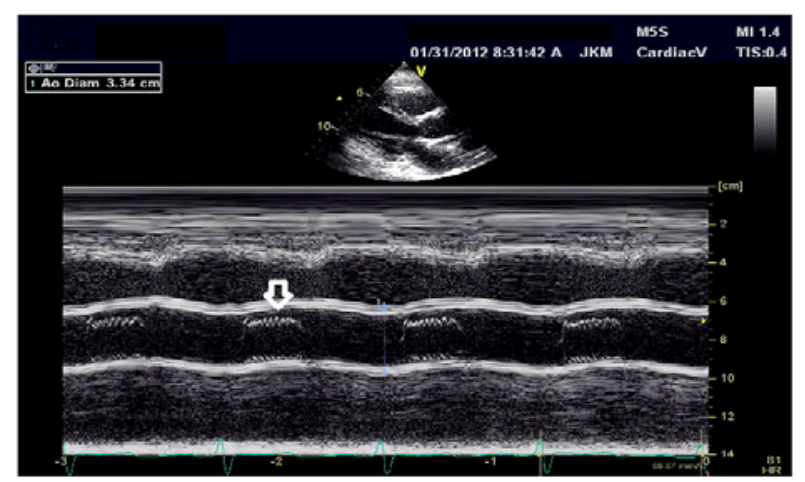

Figure 2. (b) TTE parasternal long axis view with M-mode. The white arrow indicates a finer holosystolic fluttering with decreased frequency of aortic cusps after a redo aortic root replacement. 


\section{Discussion}

We argue that patient's endocarditis was caused by high velocity jets from the Venturi effect resulting in continued damage to unprotected aortic root tubular graft with no sinuses, leading to further aortic root and aortic valve cusp lesions in a viscous cycle. As a result, a prominent high pitch murmur sound was heard across the heart precordium by physical exam throughout the systolic phase of cardiac cycle.

The Venturi effect is the reduction in fluid pressure that results when the fluid flows through a constricted section of a pipe. According to the mechanical conservation of energy, the kinetic energy has to increase in the form of higher velocity formation. This increase in velocity results in an active draw on passive structures much like air flowing with increased velocity over an airplane wing causing lift. In cardiac pathophysiology, we see Venturi effect on anterior leaflet of the mitral valve causing systolic anterior motion in patients with hypertrophic cardiomyopathy and in patients with a subvalvular ventricular septal defect in which the effect causes prolapse of the right coronary cusp resulting in aortic insufficiency ${ }^{[1]}$. Both effects are indeed velocity-induced effects on a passive leaflet or cusp.

In Figure 1a, we see the Venturi jet across the aortic valve starting in the early systolic phase. As the flow enters the prosthetic valve, velocity accelerates. This turbulent high velocity flow divides into 2 jets (white arrows) hitting the aortic root in opposite directions as seen per color Doppler pattern. Normally, the sinuses help to absorb the built-up pressure and allow the cusps to elevate, and in effect, get out of the way. In this setting, the cusps appear to be sucked into the path of blood resulting in severe turbulence and a pseudo-obstruction. Figure 2a indicates a high flow disturbance at valvular level, a phenomenon that correlates well with high frequency fluttering of aortic cusps also seen in Figure $1 \mathrm{~b}$.

The maximal aortic valve velocity recorded was $5 \mathrm{~m} / \mathrm{s}$ with the peak and mean pressure gradients of $99 \mathrm{mmHg}$ and 59 mmHg respectively per TTE apical 5 chamber view using modified and simplified Bernoulli equation $\left(\Delta \mathrm{P}=4 \mathrm{~V}^{2}\right)$, together with the calculated dimensionless index $\left(\mathrm{VTI}_{\mathrm{LVOT}} / \mathrm{VTI}_{\mathrm{AVA}}\right)$ of 0.25 , suggesting a severely reduced aortic valve area. By continuity equation $\left(\mathrm{VTI}_{\mathrm{LVOT}} \times\right.$ Area $_{\mathrm{LVOT}}=\mathrm{VTI}_{\mathrm{AV}} \times$ Area $\left._{\mathrm{AV}}\right)$, the estimated aortic valve area is $0.75 \mathrm{~cm}^{2}$ using the left ventricular outflow tract (LVOT) diameter of $1.95 \mathrm{~cm}$. Interestingly, the aortic prosthetic valve appears to open well by 2-D echo and M-mode images. At surgery under direct vision, the bioprosthetic aortic valve cusps were not only unobstructed but opened well passively as well.

We think that there are three conditions from the Venturi effect in a susceptible patient to account for this pseudoobstruction phenomenon: (1) weakening of aortic valve leaflets due to ongoing injury from a high velocity Venturi jet; (2) eccentric Venturi jets to wrap around the aortic valve cusps during systolic phase of the cardiac cycle and (3) lack of aortic root sinuses to absorb the built-up pressure leads to elevation in pressure around the area of aortic root. These two effects synergistically increase further pressure gradient between the aortic root and aortic cusp opening area, therefore leading to more obstruction.

After the redo heart surgery, TTE no longer demonstrated the turbulence of flow across the prosthetic aortic valve per 2-D color Doppler pattern. Fluttering throughout systolic cycle across the valve cusps was much finer and diminished in frequency per M-mode (see Figure 2b).

\section{Conclusion}

Given that, we hypothesize that it is the Venturi phenomenon that causes the high velocity and elevated peak and mean pressure gradient across the prosthetic aortic valve in a susceptible patient using straight tube graft with no sinuses to replace the aortic root ${ }^{[2-4]}$. The viscous cycle of further damage to aortic valve cusps and aortic root due to higher velocity Venturi jet from increasing aortic valve pseudo-obstruction makes these patients more prone to endocarditis and progressively worsening prognosis ${ }^{[5]}$. Our hypothesis is not tested, therefore still there stands no scientific evidence of our hypothesis. Moreover, staphylococcus endocarditis yet rare but well know postoperative complication after prosthetic 
aortic replacement. In one way it can also be concluded that this complication is the result of the staphylococcus endocarditis. However, it seems more logical to conclude from our work that the ventouri effect is the result of 1) the prosthetic aortic graft and 2) the presence of the endocarditis in the affected aortic valve cusps.

\section{References}

[1] Luckle M, Khattar R. Systolic anterior motion of the mitral valve-beyond hypertrophic cardiomyopathy. Heart. 2008; 94 : 1383-1385. PMid:18931154 http://dx.doi.org/10.1136/hrt.2007.122069

[2] Thubrikar M, Labrosse M, Zehr K, Robicsek F, Gong G, Fowler B. Aortic root dilatation may alter the dimensions of the valve leaflets. European Journal of Cardio-Thoracic Surgery. 2005; 28: 850-856. PMid:16275009 http://dx.doi.org/10.1016/j.ejcts.2005.09.012

[3] Burkhart H, Zehr K, Schaff H, Daly R, Dearani J, Orszulak T. Valve-preserving aortic root reconstruction: a comparison of techniques. Journal of Heart Valve Disease. 2003; 12: 62-67. PMid:12578338

[4] Sternik L, Zehr K, Schaff H. A method of repair for asymmetric aneurysmal dilatation of the ascending aorta. Annals of Thoracic Surgery. 2002; 73: 1332-1334. http://dx.doi.org/10.1016/S0003-4975(01)03457-9

[5] Zehr K, Orszulak T, Mullany C, Matloobi A, Daly R, Dearani J, et al. Surgery for aneurysms of the aortic root: a 30 -year experience. Circulation. 2004; 110: 1364-1371. PMid:15313937 http://dx.doi.org/10.1161/01.CIR.0000141593.05085.87 